\title{
A rare case of uterine perforation due to gas gangrene of uterus caused by Clostridium Perfringens
}

\author{
Poonam Kashyap, Sudha Prasad, C B Singh \\ Correspondence: Dr Poonam Kashyap, Department of Obstetrics and Gynaecology, \\ Maulana Azad Medical College and Lok Nayak Hospital, Delhi, India; Email - \\ drpoonamkashyap@gmail.com
}

Distributed under Creative Commons Attribution-Share Alike 4.0 International.

\begin{abstract}
Clostridium Perfringens infection is an extremely fatal condition which is difficult to diagnose in first instance. A 65 year old lady para 3 , presented with acute abdominal pain and vomiting to the surgical emergency. She was admitted,investigated and kept on conservative management. Her blood investigations, CT scan findings suggested bowel perforation. On laparotomy, acute gas gangrene infection of uterus was found leading to myometrial necrosis and perforation. Hysterectomy was performed and patient started on antibiotics but patient did not improve in postoperative period and expired. On histopathology, sepsis with gas gangrene was confirmed. Clostridium Perfringens infection affecting primarily uterus is a very rare so there is need to spread the awareness of this infection to prevent mortalities because of this infection.
\end{abstract}

Keywords: Clostridium Perfringens, uterine perforation, gas gangrene.

Clostridia are gram positive bacilli and are normally present in human intestine and vaginal floara in 1 to $27 \%$ of healthy female. ${ }^{1,2}$ The ascending infection can occur due to any interventions like septic abortions. It is also found in 10 to $27 \%$ of patients undergoing elective abortions. ${ }^{2}$ This infection is very rare and extremely fatal especially in nonpregnant uterus and not many cases are reported. Few cases have been reported to be associated with uterine malignancy. All patients present with acute pain abdomen, vomiting and fever.

The differential diagnosis could be acute perforation of appendix, acute cholecystitis, acute pancreatitis. This article describes the rare case of nonpregnant woman with clostridium infection leading to mortality inspite of prompt resuscitation, intraoperative diagnosis and aggressive surgical treatment.

\section{Case Report}

A lady of 65 years of age with para 3 status presented with acute pain abdomen and vomiting of 10 hours duration. Patient didn't give history of fever though she was febrile on admission. She was known hypertensive on regular treatment. There was no past history of diabetes, thyroid disease, other medical disorder or any surgery.

Patient did not have pallor but appeared in distress because of abdominal pain. She had pulse rate of $110 / \mathrm{min}$, $\mathrm{BP}$ was $110 / 76 \mathrm{~mm}$ of $\mathrm{Hg}$, respiratory rate was $22 / \mathrm{min}$ and oxygen saturation was $98 \%$. Chest and cardiovascular examination did not reveal any abnormality. Abdominal examination revealed distension with rebound tenderness on palpation.

Received: 5 th June 2017. Accepted: 12 th August 2017.

Kashyap P, Prasad S, Singh CB. A rare case of uterine perforation due to gas gangrene of uterus caused by Clostridium Perfringens. The New Indian Journal of OBGYN. 2018; 4(2): 165 - 67. 
Her blood picture showed leucocytosis $(13,600)$ with predominant neutrophils. Arterial blood gas analysis showed metabolic acidosis. The urine output of the patient was decreased. Her X-ray and CT scan of abdomen showed free gas in abdomen. With the diagnosis of acute perforation peritonitis patient was taken for urgent exploratory laparotomy.On opening peritoneal cavity a large gush of foul smelling free gas came out. Further exploration showed an area of sloughed out anterior uterine wall with $2 \mathrm{~cm}$ perforation in the centre (Figure 1). Anterior wall was covered with exudate and blebs. There was myometrial gangrene involving tubes and ovaries were also acutely inflamed. There was copious exudative fluid on and around uterine surface. The small and large bowel was found to be normal. Hysterectomy with bilateral salpingoophorectomy was done. Peritoneal lavage was done and drain was left in abdominal cavity. The abdomen was closed with retention sutures. Peritoneal fluid cultures were sent and hysterectomy specimen was sent for histopathological examination. Patient had severely decreased urine output despite adequate fluid rescucitation, developed end organ damage. She was intubated and shifted to intensive care unit. She was kept on Piperacillin/Tazobactum and metronidazole intravenous antibiotic. On postoperative day 1 , patient was severely acidaemic and developed hypotension. She was started on norepinephrine for pressure support. There was copius drainage of pus from drain. She had progressive end organ damage with distension of abdomen noticed on day 4 . The condition of the patient deteriorated further and she expired on postoperative day 6 due to shock following sepsis and multi organ failure. On histopathology, sepsis with gas gangrene was confirmed.

\section{Discussion}

Till date, only 18 cases of uterine infection with gas gangrene have been reported and out of this only 6 case have been reported in non pregnant uterus. This case is the $7^{\text {th }}$ documented case of clostridium induces myometrial necrosis in non pregnant uterus. ${ }^{1}$

Clostridium Perfringens is encapsulated gram positive anaerobic, non motile bacilli and is capable of producing exotoxins. One of the alpha toxins, Lecithinase $C$ causes intravascular hemolysis leading to anaemia and jaundice and it may cause intravascular hemolysis as a result of its necrotizing action on capillaries and other blood vessels. ${ }^{2,3}$ Infection with Clostridium rarely result in septicaemia. The onset of symptoms from time to infection is rapid 12 to $24 \mathrm{hrs}$. In case of septicaemia, there will be clinical findings of shock. The patient will have acute hemolysis, fast progression of jaundice, anaemia, tachycardia, hypotension and may lead to death. The mortality rate is from 50 to $85 \%$ which is very high. ${ }^{1}$ There are 640 strains of Clostridium Welchii known, out of which only $5 \%$ are virulent. The ideal conditions for the bacteria to grow are decrease in $\mathrm{pH}$ and decrease in oxygen tension. Therefore necrotic and injured tissue

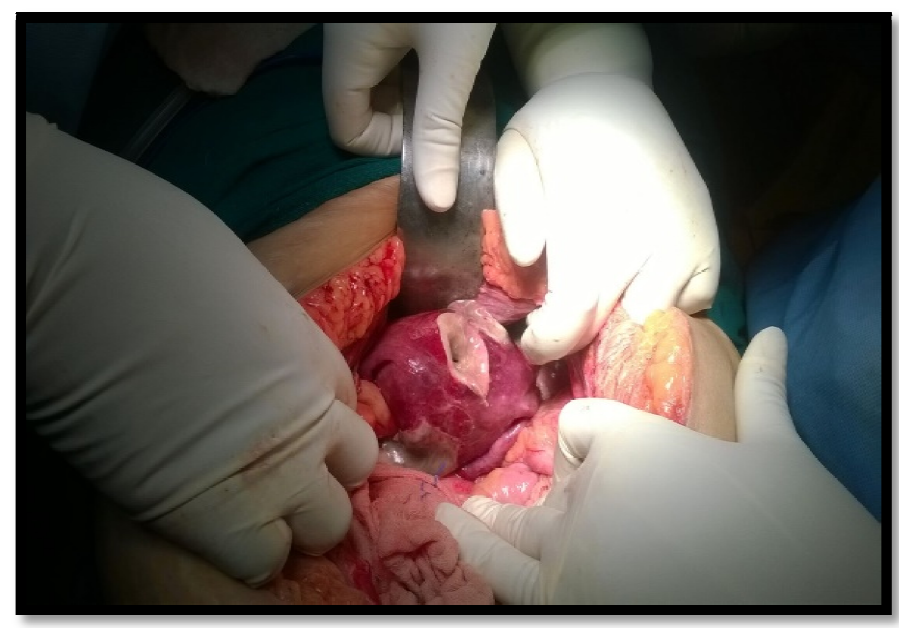

Figure 1: Showing perforation in sloughed out anterior uterine wall (marked by red thick arrow). Rest of uterus shows pregangrenous changes

permits the growth of bacterial colonies and production of toxins. That is why this infection is more commonly seen after deliveries, abortions, molar pregnancies, and in

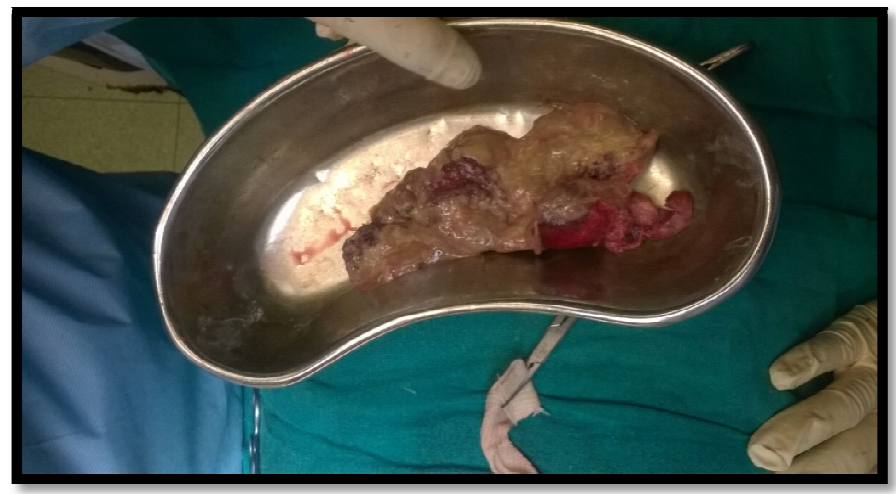

Figure 2: Specimen showing sloughed 
patients with underlying malignancies. ${ }^{4}$ In presence of malignancies, rapid turnover of cells provides hypoxemic and ischaemic environment permitting the growth of bacteria. $^{5}$

C. Perfringens septicaemia leading to gas gangrene has high mortality. It requires prompt diagnosis and treatment to prevent mortality. Therefore, this article emphasises the importance of early detection and to have clinical suspicion when the clinical findings are not corroborating to any of the more common causes of shock. Its rapidity of onset, presence of shock in the absence of any clear cardiac, pulmonary or renal pathology should alert the physician to suspect C. Welchii septicaemia. Prompt diagnosis and immediate surgery to remove the affected site, treatment with antibiotics and appropriate fluid management can reduce the mortality in $\mathrm{C}$. Welchii sepsis from 50 to $80 \%$ to 10 to $20 \%$. Penicillin group of antibiotics are used in its treatment. Antibiotics alone are not sufficient and surgical debridement may be required to remove affected tissue in some cases. ${ }^{6}$

\section{Conflict of interest: None. Disclaimer: Nil.}

\section{References}

1.David K, Nagarajan M, Benjamin C, Fidencio D, Michael B, Akella C. Uterine Perforation with Intra-Abdominal
Clostridium perfringens Gas Gangrene: A Rare and Fatal Infection. J Of Gynaecol Surg. 2016; 32:182

2.Kurashina R, Shimada H, Matsushima T, Doi D, Asakura H, Takeshita T. Spontaneous uterine perforation due to clostridial gas gangrene associated with endometrial carcinoma. J Nippon Med Sch. 2010; 77: 166.

3.Nakamura M, Cross WR. The lecithinase (alpha toxin) activity of strains of Clostridium perfringens. Proc Soc Exp Biol Med. 1968; 127: 719.

4.Browne JT, Van Derhor AH, McConnell TS, Wiggins JW. Clostridium perfringens myometritis complicating cesarean section: Report of 2 cases. Obstet Gynecol. 1966; 28: 64.

5.Larson CM, Bubrick MP, Jacobs DM, West MA. Malignancy, mortality, and medicosurgical management of Clostridium septicum infection. Surgery. 1995; 118: 592.

6.Myers RAM. Hyperbaric oxygen therapy in clostridial infection. South Med J 1985; 78: 227.

Poonam Kashyap ${ }^{1}$, Sudha Prasad ${ }^{2}$, C B Singh ${ }^{3}$

${ }^{1}$ Gynaecologist, ${ }^{2}$ Gynaecologist; Department of Obstetrics and Gynaecology, Maulana Azad Medical College and Lok Nayak Hospital, Delhi; ${ }^{3}$ Surgeon, Department of Surgery, Maulana Azad Medical College and Lok Nayak Hospital, Delhi, India 